\title{
Membrane-based Separation in Flow Analysis for Environmental and Food Applications
}

\author{
Inês C. Santos, Raquel B. R. Mesquita \& António O. S. S. Rangel
}

To cite this article: Inês C. Santos, Raquel B. R. Mesquita \& António O. S. S. Rangel (2020)

Membrane-based Separation in Flow Analysis for Environmental and Food Applications, Separation \& Purification Reviews, 49:1, 37-54, DOI: 10.1080/15422119.2018.1506810

To link to this article: https://doi.org/10.1080/15422119.2018.1506810

册 Published online: 20 Aug 2018.

Submit your article to this journal $\widetilde{ }$

山ll Article views: 158

Q View related articles $\asymp$

View Crossmark data 


\title{
Membrane-based Separation in Flow Analysis for Environmental and Food Applications
}

\author{
Inês C. Santos, ${ }^{1}$ Raquel B. R. Mesquita, and António O. S. S. Rangel \\ Universidade Católica Portuguesa, CBQF - Centro de Biotecnologia e Química Fina - Laboratório \\ Associado, Escola Superior de Biotecnologia, Rua Arquiteto Lobão Vital, 172, Porto, Portugal
}

\begin{abstract}
Membrane-based separation techniques have been used as an efficient process for analyte separation or enrichment and matrix removal. By coupling these techniques to flow-based analysis, sample preparation and analyte detection can be automated and miniaturized. Different membrane separation techniques are available but the most used in flow analysis are gas diffusion, dialysis, supported liquid membranes and polymer inclusion membranes. The current state of the art of membrane-based separations hyphenated with flow techniques is presented along with a discussion of the applications to environmental and food analysis. Moreover, a brief description of gas diffusion, dialysis and membrane extraction techniques is also included.
\end{abstract}

Keywords: Membrane-based separation techniques, flow analysis, gas diffusion, dialysis, supported liquid membranes, polymer inclusion membranes

\section{INTRODUCTION}

Sample pre-treatment is one of the bottlenecks of analytical chemistry and because of that it has received special attention. Different sample pre-treatment techniques such as liquidliquid extraction (LLE), solid-phase extraction (SPE), membrane-based separation and digestion have been used (1-3). However, when performed in a batch mode these methods are tedious and time consuming (4). In order to improve the sample throughput and the method precision, sample pre-treatment methods have been coupled to flow analysis techniques (5-10). Figure 1 shows a timeline of the flow injection techniques and Figure 2 their representation (11-14).

The first generation of flow analysis was described in 1975 by Ruzicka and Hansen and is based on a continuous flow where the need to achieve chemical and physical equilibrium,

Received 22 March 2017, Accepted 3 July 2018.

Address correspondence to António O. S. S. Rangel, CBQF - Centro de Biotecnologia e Química Fina - Escola Superior de Biotecnologia, Universidade Católica Portuguesa, Rua Arquiteto Lobão Vital, 172, Porto 4200-374, Portugal. E-mail: arangel@porto.ucp.pt

${ }^{1}$ Present address: Department of Chemistry and Biochemistry, The University of Texas at Arlington, Arlington TX, USA

Color versions of one or more of the figures in the article can be found online at www.tandfonline.com/lspr. a practice in batch analysis, was discarded. Flow injection analysis (FIA), as it was called, consists in the injection of a well-known volume of sample in a flowing stream of carrier/ reagent. Different reagents can be added by means of confluences and the mixing occurs while the sample is propelled toward the detector. This technique provides the analysis of several samples in an automatic way using a simple manifold with high throughput. In spite of its significant advantages, FIA also presents some disadvantages such as the high sample and reagents consumption and the need for physical reconfiguration in multi-parametric analysis.

To overcome these limitations, sequential injection analysis (SIA) was described in 1990 by Ruzicka and Marshall (12). This technique is based on a programmable and discontinuous flow, controlled by computer. Sample and reagents are sequentially aspirated and mixing occurs by flow reversal toward the detector. Due to the programmable flow in SIA, reagents are only pumped and consumed when needed, so lower reagents and sample volumes are used, which decrease the consumption values and waste production. Also, as a multiposition valve is used instead of an injection valve, the coupling of different reagents, detectors and devices is possible without the need for manifold reconfiguration. Therefore, multi-parametric determinations are more easily performed using SIA (15). 


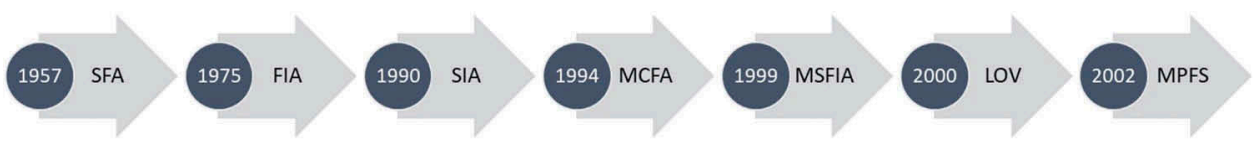

FIGURE 1. Timeline of the flow analysis techniques.

Meanwhile, other flow techniques were also described namely micro-sequential injection lab-on-valve ( $\mu$ SI-LOV), multicommuted flow analysis (MCFA), multisyringe flow injection analysis (MSFIA) and multipumping flow systems (MPFS) $(13,14)$. All these flow techniques, including FIA and SIA, are based on the same principles: (i) reproducible sample injection; (ii) controlled dispersion of the sample zone and (iii) reproducible timing of its movement from the injection valve to the detection system. Flow techniques are effective tools for analytical chemistry as they can be used for the determination of a wide range of analytes in a fast and automatic way. Moreover, they can also be used for the following purposes: (i) sample introduction into the detector; (ii) automation of sample pre-treatment; (iii) sample dispersion/dilution and (iv) analyte derivatization $(11,15,16)$. In fact, flow techniques can be coupled with almost any detection device, which allows the detector to be exposed to the sample only for brief periods of time minimizing potential damage or contamination. Furthermore, the conditioning, cleaning and calibration of the detector are effectively performed.

When analyzing complex samples, some challenges may be encountered due to sample matrix interferences and to low analyte concentration (i.e., trace levels). Coupling flow techniques with separation devices enables the collection, enrichment and separation of the analyte from the matrix prior to detection. Therefore, an automation of sample pre-
A)
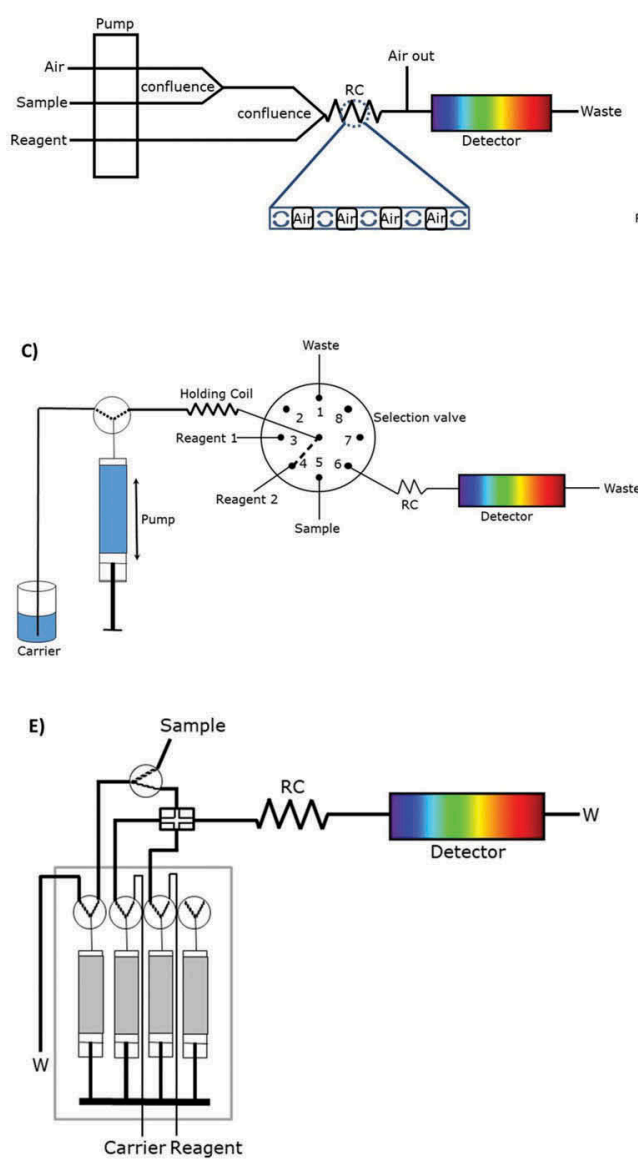

B)

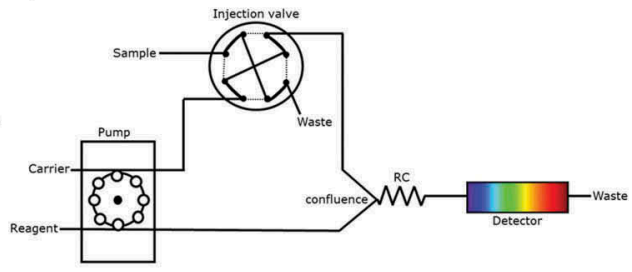

D)

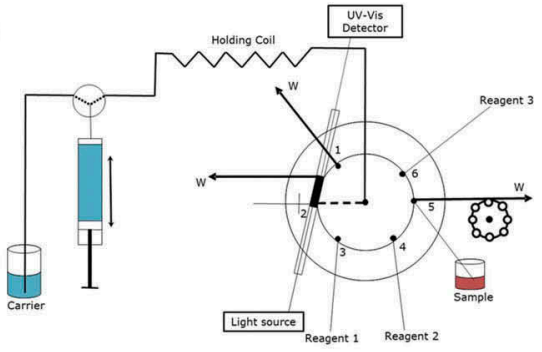

F)

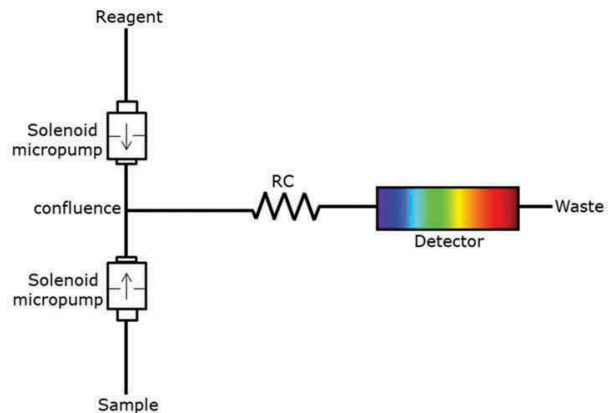

FIGURE 2. Schematic representation of different flow analysis manifolds: (A) segmented flow analysis; (B) flow injection analysis; (C) sequential injection analysis; (D) sequential injection lab-on-valve; (E) multisyringe flow injection analysis; (F) multipumping flow analysis (11-14). 
treatment is achieved with a decrease in sample and reagents consumption. As previously shown by Santos et al. (17), SPE is the most used separation method in flow analysis. Nevertheless, membrane-based separations are still preferred to LLE and digestion (18). An overview of the membrane-based separation techniques and their use in flow analysis is presented in the following sections.

\section{MEMBRANE-BASED SEPARATIONS}

Membrane-based separation techniques have been used as an alternative to SPE and LLE as they are cheaper and easier to perform $(19,20)$. In membrane-based separation techniques, a membrane (in a solid or liquid state) is used to separate two phases while the components are transferred from a donor in one phase to an acceptor solution in the other phase. This transfer can be achieved by means of different driving forces such as concentration differences, electric potential difference and pressure difference (21). Moreover, the diffusion of the analyte can be of three types: passive, facilitated and active. In passive diffusion, the components diffuse under the influence of a gradient. In facilitated and active diffusion, a carrier is placed in the membrane to increase the membrane permeability and therefore facilitate the diffusion of the analyte. Nevertheless, in facilitated diffusion, the analyte diffuses from high to low concentration gradients while, in active diffusion, the analyte diffuses against the gradient through a reaction that occurs inside the membrane (22).

Within membrane-based separations and according to the morphology and porosity of the membrane used, different techniques such as gas diffusion (GD), dialysis and liquid membrane extraction can be performed.

\section{Gas Diffusion}

GD is used for separation of volatile components that can occur either in the gas phase or in aqueous solutions. A hydrophobic membrane is used to separate the donor from the acceptor phase where the non-selective permeation of gaseous analytes occurs. As only few compounds are volatile at room temperature, GD has a high degree of selectivity since, for example, ionic compounds are excluded. First, the analyte evaporates into the membrane pores and afterward, the volatilized analytes diffuse through the membrane and are absorbed by the flow of an aqueous solution on the acceptor channel. Chemical species such as dissolved carbon dioxide and dissolved oxygen can be separated using a GD unit. Furthermore, the applicability of this technique can be increased converting aqueous species into gases by addition of an acid or alkaline solution to the sample in the donor phase. The addition of an acid solution to the donor phase may release gases such as carbon dioxide, hydrogen sulfide and chlorine from carbonate, sulfate and hypochloride, respectively, while the addition of an alkaline solution to the donor phase can, for example, convert ammonium ions into ammonia gas (20-23).

The driving force in GD membrane separation is the concentration gradient and the diffusion efficiency is mainly governed by the Fick's law (23). However, the diffusion of a species can also be influenced by the membrane material, membrane path length and area, membrane porosity and membrane thickness. To improve the diffusion efficiency, the membrane path length and area should be as large and as thin as possible, to result in a large contact area easy to cross. However, a compromise between mass-transfer efficiency and membrane strength and lifetime may be required.

In GD, derivatizing reactions can be carried out in the acceptor stream for two main reasons: to help maintain the concentration gradient thereby increasing the rate of diffusion or to form detectable derivatives for detection (23).

\section{Dialysis}

Dialysis has been thoroughly described in previous reviews (23-25), which demonstrate its importance in sample pretreatment. According to the driving forces used, this technique can be referred to as passive dialysis, Donnan dialysis and electrodialysis. The most widely used approach is the passive dialysis, where the analyte is separated from liquid samples by diffusion through a hydrophilic membrane according to its molecular weight. The passive dialysis is a good ultrafiltration molecular filter as it allows small molecules and ions to pass through the membrane, while macromolecules are held in the donor stream. Due to these reasons, this technique is not considered a selective method for separation and is mainly used to perform sample dilution. In Donnan dialysis or active dialysis, ions are transferred through an ion exchange membrane due to the ionic strength gradient while in electrodialysis, the analyte is separated by an electrical field (26).

Dialysis is not usually used as an extraction technique per se due to the lack of discrimination. Therefore, it is usually used as an additional cleanup step. Nevertheless, it has been widely applied to food and biological samples for desalting, buffer exchange, removal of labelling reagents, drug binding, cell growth and feeding, virus purification and blood treatment since macromolecules are excluded from passing through the membrane. Derivatizing reactions can also be carried out in the acceptor stream of the dialysis unit for the same reasons as the ones previously stated in GD (23).

\section{Membrane Extraction}

Membrane extraction is a separation technique that uses mainly nonporous membranes, in a liquid or solid state (polymer impregnated with a liquid) that are placed between two liquid phases. Membrane extraction includes different approaches such as supported liquid membrane extraction (SLME), microporous membrane liquid-liquid extraction (MMLLE), polymeric membrane extraction (PME) or 
polymer inclusion membranes (PIMs) and membrane extraction with a sorbent interface (MESI) $(27,28)$. Of these approaches, in this manuscript, we will mainly discuss SLME and PIMs for membrane extraction as these are the most used in flow analysis for environmental applications.

\section{Supported Liquid Membranes}

Supported liquid membrane (SLM) is the most important liquid membrane technique and was developed to enhance the diffusion through the membrane. As the diffusion coefficient is higher in liquids than in solids, the diffusion is enhanced in SLMs when compared to dialysis. The SLM operates with two aqueous phases, the donor and acceptor solutions, separated by an organic phase, the liquid membrane, impregnated in the pores of a polymeric support by capillary action. Therefore, in SLM, two LLEs are carried out sequentially: first, the analytes are extracted from the aqueous sample to the organic phase in the membrane and afterward they are extracted from the membrane to the aqueous acceptor phase. In fact, this separation technique is a good alternative to LLE as it has a similar procedure but with significant advantages such as easier automation and operation, and lower organic solvent consumption (28).

In SLMs, reactions in the donor phase can convert the analyte into a non-ionic form therefore favoring mass transfer. Once in the acceptor phase, the non-ionic form must be converted back into its ionic form that is irreversibly trapped in this phase. Derivatizing reactions can also be performed in the acceptor stream with the same purposes as for GD and dialysis.

The molecules separated by SLM are mainly highly polar ionic or ionizable compounds such as organic acids and bases, charged compounds and metal ions. To improve the analyte permeability and selectivity, the addition of a complexing agent to the liquid membrane to act as carrier can be done to react selectively and reversibly with the analyte. In this way, facilitated or coupled transport processes can be performed.

\section{Polymer Inclusion Membranes}

Recently, a new approach, named polymer inclusion membrane $(29,30)$ was developed for the liquid separation of metal ions and small organic molecules. This approach is a more stable, versatile and selective alternative to SLMs. In PIMs the carrier is entrapped in the membrane matrix while in SLMs the carrier containing solvent is impregnated on a porous polymer film. PIMs consist of a base polymer, usually poly(vinyl chloride) (PVC) or cellulose triacetate (CTA), a carrier for analyte extraction and transport and a plasticizer. The separation procedure occurs in the same way as in SLMs. The main advantage of PIMs is its high selectivity as they are prepared according to the intended analyte.

The principles of separation previously described for GD, dialysis, SLM and PIM techniques are schematically shown in Figure 3. A summary of the most important features of each technique is also presented in Table 1.

As previously explained, in GD and dialysis (Figure 3 I and II), the diffusion occurs by concentration gradient. In SLME (Figure 3 III), the solute must be in two forms, nonionic in the donor phase and ionic in the acceptor phase, where it is irreversibly trapped. This can be achieved by adjusting the $\mathrm{pH}$ in both phases. The separation by SLMs can also be achieved by adding a carrier to the liquid membrane further enhancing the diffusion which resembles the separation with PIMs (Figure 3 IV).

Different forms of membrane separation units such as tubular (or hollow fiber) and sandwich can be used. Hollow fiber and spiral units increase the surface area and therefore enhance the diffusion of the analyte. Furthermore, when using the sandwich unit, different grooves (spiral, rectangular and winding) can be explored to improve the diffusion efficiency (23).

\section{MEMBRANES - PHYSICOCHEMICAL STRUCTURES AND FEATURES}

Different membranes have been used for on-line sample preparation however not yet to their fully extent. Membranes are differentiated based on their pore size, microstructure (homogeneous, microporous, fibrous), physicochemical properties (hydrophilic and hydrophobic), reactivity and shape.

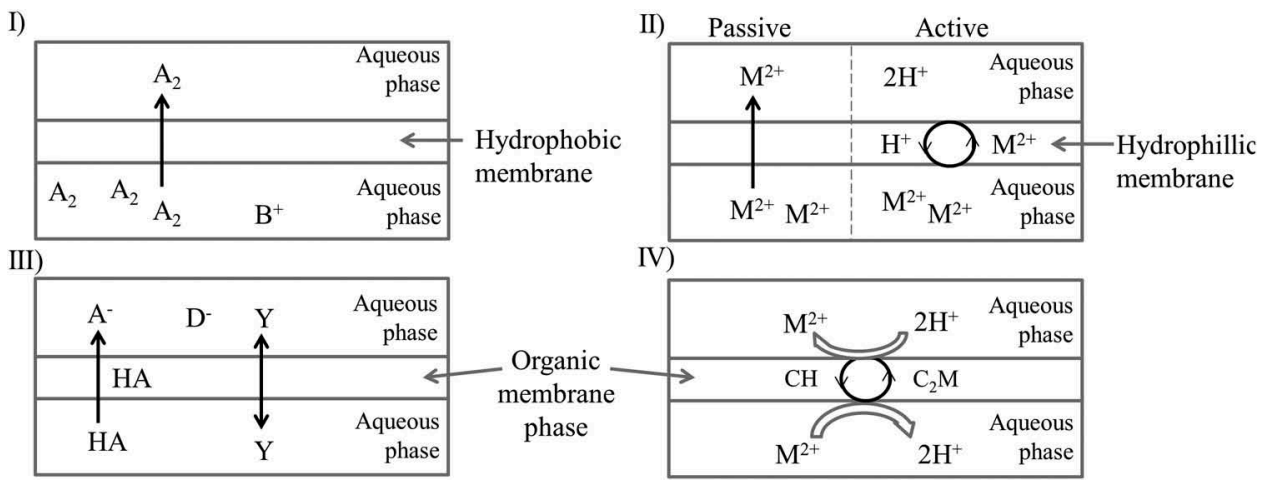

FIGURE 3. Schematic representation of the membrane extraction processes in: (I) gas diffusion; (II) dialysis; (III) supported liquid membrane and (IV) supported liquid membrane or polymer inclusion membrane (the carrier is represented by the letter C) (23-28). 
TABLE 1.

Features of membrane-based separation processes (19)

\begin{tabular}{|c|c|c|c|c|}
\hline $\begin{array}{l}\text { Membrane-based } \\
\text { separation }\end{array}$ & Analyte(s) & Driving force & Phases (Donor-membrane-acceptor) & Membrane material \\
\hline Gas diffusion & Volatile compounds & $\begin{array}{l}\text { Analyte pressure gradient } \\
\text { (concentration gradient) }\end{array}$ & Aqueous-gas-aqueous & $\begin{array}{l}\text { Hydrophobic and } \\
\text { microporous }\end{array}$ \\
\hline $\begin{array}{l}\text { Dialysis } \\
\text { (passive and active) }\end{array}$ & $\begin{array}{l}\text { Low molecular weight species } \\
\text { and ions }\end{array}$ & $\begin{array}{l}\text { Concentration gradient } \\
\text { (passive dialysis) } \\
\text { Ionic strength gradient } \\
\text { (active dialysis) }\end{array}$ & $\begin{array}{l}\text { Aqueous-aqueous (passive dialysis) } \\
\text { Aqueous-membrane-aqueous (active } \\
\text { dialysis) }\end{array}$ & $\begin{array}{l}\text { Hydrophilic and } \\
\text { microporous (passive } \\
\text { dialysis) } \\
\text { Ionic and microporous } \\
\text { (active dialysis) }\end{array}$ \\
\hline SLMs & $\begin{array}{l}\text { Organic acids and bases, } \\
\text { charged compounds and }\end{array}$ & $\begin{array}{l}\text { Change of partition } \\
\text { coefficient }\end{array}$ & Aqueous-organic-aqueous & $\begin{array}{l}\text { Hydrophobic and } \\
\text { homogeneous }\end{array}$ \\
\hline PIMs & metal ions & Partition coefficient & $\begin{array}{l}\text { Aqueous-polymer-aqueous or organic- } \\
\text { polymer-aqueous or aqueous-polymer- } \\
\text { organic }\end{array}$ & $\begin{array}{l}\text { Hydrophobic and } \\
\text { homogeneous }\end{array}$ \\
\hline
\end{tabular}

Microporous membranes are structurally similar to a conventional nanofiltration filter (31) and consist of a solid matrix with defined holes which have diameters from 1-10 nm. Typically, microporous membranes are made of cellophane, cellulose acetate, polycarbonate, polysulfone, polyacrylonitrile, polyamide and others. Separation is achieved solely by a sieving mechanism and so pore diameters and molecule sizes are the determining parameters. In this way, a membrane with smaller pores favors the diffusion of small molecules impairing the passage of macromolecules such as colloids. Their structure may be symmetric or asymmetrical (31).

Homogeneous membranes consist of a dense film through which a mixture of molecules is transported by diffusion under the driving force of a pressure, concentration or electrical potential gradient. The separation of various components of a mixture is related directly to their relative transport rates within the membrane, which are determined by their diffusivity and solubility in the membrane phase. Most gas separation, pervaporation and reverse osmosis processes use homogeneous membranes to perform the separation. Homogeneous membranes are usually made of polymers (31).

Electrically charged membranes are highly swollen gels carrying fixed positive (anion-exchange membranes) or negative charges (cation-exchange membranes) where the charged groups are fixed to the polymer. The main application of ion-exchange membranes is in electrodialysis.

Liquid membranes utilize selective carriers to transport certain molecules at a relatively high rate. These can be made by filling a microporous polymer structure with a liquid membrane phase.

Most membranes are synthetic organic polymers (e.g., polysulfone, cellulose acetate) but can also be prepared from inorganic materials such as ceramics or metals. Polymeric membranes are economical and technologically useful; however, their performance is limited and their permeability must be sacrificed for selectivity and vice versa. Polymeric porous membranes are widely used in SLMs and polymeric non-porous membranes in PIMs. Most synthetic polymeric membranes are generally hydrophobic, therefore, hydrophilic additives such as polyvinylpyrrolidone (PVP) are included to make membranes hydrophilic.

For dialysis, hydrophilic membranes of microporous, homogeneous and ion-exchange types are used, usually made of cellulose acetate or similar materials (e.g., Cuprophane or Cellophane). They need to be hydrated by an overnight stay in water before use. For GD, hydrophobic membranes (e.g., PTFE or PVDF membranes) are used and for SLMs a porous PTFE solvent-filled membrane is used (32).

Only a handful of materials are used to make commercial membranes since, in addition to permeability and selectivity, the products must be inexpensive and stable to allow reproducible data. Both dialysis and GD membranes are cheap and easy to obtain as they are extensively used in industry. Work is currently being performed to develop new membrane materials of higher sensitivity and permeability.

\section{MEMBRANE-BASED SEPARATIONS HYPHENATED TO FLOW TECHNIQUES}

Membrane-based separation techniques are fairly easily implemented in flow analysis and they have been explored mainly due to advantages such as increased throughput, compatibility with most of the detection techniques, possibility to separate sample matrix and analyte enrichment. In fact, the ability to automate and miniaturize the sample preparation procedure, potentially increasing the precision of the method, is the driving force stimulating the mentioned combination. Additionally, in flow techniques the mass transfer does not need to reach equilibrium as it is required in a batch mode, which allows a faster determination rate. However, due to this reason it is vital to control the operation time. 
As previously stated, membrane-based separation processes in flow analysis can be used in sandwich or tubular modes. Sandwich mode with a flat membrane is the most frequently used, since it is easy to mount and is widely available. The tubular membranes are difficult to mount and the outer chamber usually has a larger dead volume which is considered a drawback. In both approaches the devices can be coupled to different flow techniques such as continuous flow, FIA, and SIA at different locations (23). They can be placed in a pre-valve setup, either before or after the peristaltic pump, inside the loop of the injection valve, between the injection system and the detector or as part of the detector. The latter configuration already exists as a commercial product developed by FIAlab named "sandwich gas membrane sensor" (33). In this commercial product, the item includes both separation and detection. The analyte is measured as it is being diffused from the membrane and so less dilution occurs and a higher sensitivity can be obtained.

When the membrane device is placed in the injection valve, the acceptor phase is stopped for analyte enrichment and afterward is injected toward the detector. The pre-pump and pre-valve configurations can be an appropriate approach to perform sample dilution or to clean samples containing suspended solids. Nevertheless, the most used configuration is the one that places the membrane unit between the injection system and the detector. The separation process can be accomplished by continuously pumping the sample and by keeping the acceptor phase either continuously pumping or stopped. The last strategy may result in increased sensitivity and less analyte dilution as the separation unit can be placed near the detector. Derivatizing reactions in the acceptor channel are also a way to improve the diffusion efficiency as previously explained.

A search for the published papers was made on ISI Web of Knowledge - Web of Science, time span 2000-2016 describing the hyphenation of membrane-based separations with flow techniques. The distribution of the scientific literature in this field is shown in Figure 4.

As previously mentioned, membrane separation processes facilitate analyte enrichment and separation from the matrix minimizing potential interferences. When compared to SPE and LLE, membrane-based separation units are an interesting alternative for sample preparation as they are cheaper to obtain and easily coupled to flow techniques with lower organic reagent consumption. However, their utilization in flow analysis has been occasional (Figure 5). Nevertheless, of the membrane-based extraction techniques discussed, GD is the most used in flow analysis. In fact, this technique is more selective than dialysis and simpler to perform than membrane extraction techniques. Few publications exist describing the use of PIMs in flow analysis as it is a fairly recent technique.

The determination of chemical species in both environmental and food matrices presents unique analytical challenges, namely ultra-low concentrations and variable composition and complexity of the sample matrices. The use of flow methods hyphenated with membrane-based extraction offers many attractive features for addressing these challenges. As previously described, the automation and miniaturization of the sample preparation procedure is possible with higher throughput together with analyte enrichment and matrix interferences minimization. Consequently, although the flow-based membrane extraction techniques can be widely applied, the main areas of application are food and environmental analysis (Figure 6). Most of the applications to food analysis have been performed using SIA while the application to environmental analysis has been equally performed using FIA and SIA. Combining membrane separation devices with SIA uses the same principles as coupling with FIA but with substantially reduced sample and reagent consumption.

A search of the papers describing the use of membranebased extraction techniques in flow analysis for environmental (Table 2) (34-60) and food analysis (Table 3) (61-98) since the year of 2000 was made. In environmental analysis, naturally occurring gaseous species (e.g., carbon dioxide, chlorine dioxide and sulfur dioxide) or easily converted species (e.g., ammonium, dissolved inorganic carbon, sulfite and bromide) are the most targeted using FIA-GD. These determinations can be achieved with increased selectivity even in complex environmental samples. Successful applications of this hyphenation can be observed in: the determination of bromide and sulfide in tap and wastewater by spectrophotometry $(50,55)$; chlorine dioxide in water by fluorescence $(47)$; and ammonia and inorganic carbon in water with an acidbased indicator by spectrophotometry $(42,44)$, conductimetry $(36,38,56,58)$ and fluorescence $(41,49)$. Due to the high selectivity of GD membranes, most of the methods combine membrane-based separations with non-selective detectors such as spectrophotometric and conductimetric detection where the color change of acid-base indicators or conductivity changes are measured (36,38,40,42,44,48,50,54$56,58,59)$. Fluorescence and chemiluminescence detectors are usually chosen when lower detection limits are intended as these are sensitive techniques $(37,39,41,43,47,49)$.

In food analysis, FIA-GDU has also been used for isolation of volatile analytes and matrix elimination. Usually, food samples have a complex matrix presenting intrinsic absorption which can interfere with the detection and therefore it's elimination is necessary prior to analyte detection. Furthermore, some analytes are expected to be present at high concentration values and a dilution may be needed, which can also be attained with the FIA-GDU technique. Different methodologies using FIA-GDU have been described for the determination of ethanol either by colorimetric determination (77) or based on the schlieren effect (refractive index changes and/or optical variations) (94). In the former work, ethanol is collected into the acceptor stream containing acidic dichromate solution which leads to the formation of green $\mathrm{Cr}(\mathrm{III})$, monitored at $600 \mathrm{~nm}$. In 


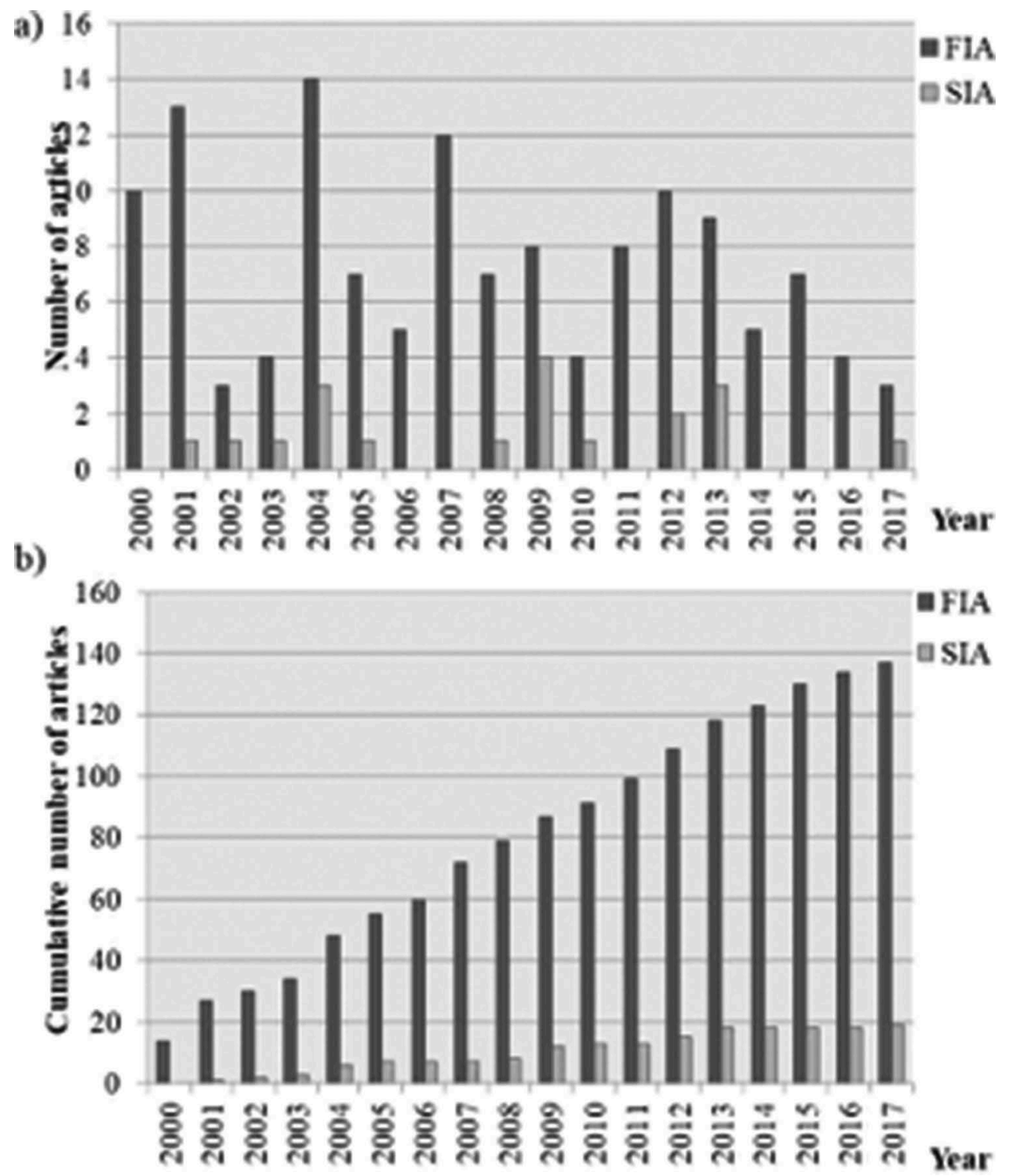

FIGURE 4. Distribution of the membrane-based flow analysis papers in the timespan 2000-2017; a, count per year; b, cumulative count (search made on ISI Web of Knowledge - Web of Science in October $25^{\text {th }} 2017$ ).

the later work, ethanol diffuses through the membrane and the schlieren effect caused by this compound in the acceptor stream is measured without the need of using reagents. Additionally, GDU can be used to separate volatile compounds originated from enzymatic reactions. In the work by Iida et al. (79) an acid urease column was used for the conversion of urea in carbon dioxide that was consequently diffused through a GDU and determined spectrophotometrically following the color change of a $\mathrm{pH}$ indicator.

SIA has also been coupled to GDU (SIA-GDU) for the determination of volatile analytes $(37,39,40,48,59,66,70$ $72,75,82,96,98)$ in both food and environmental analysis. In the work by Pais et al. (96), a sequential injection system was developed for ethanol determination using the GDU to perform in-line dilution. The detection was based on the spectrophotometric enzymatic reaction catalyzed by alcohol dehydrogenase in the presence of nicotinamide adenine dinucleotide $\left(\mathrm{NAD}^{+}\right)$. In the work by Segundo et al. (40), an environmental application of SIA-GDU is described for the determination of ammonium in water samples. Ammonium is converted to ammonia by adding sodium hydroxide in-line. Afterward, ammonia diffuses through the GDU for matrix separation and changes the color of a $\mathrm{pH}$ indicator present in the donor channel. This color change is monitored spectrophotometrically.

Dialysis has been more explored for food analysis and therefore fewer works describe environmental applications $(52,53)$. This may be due to the dialysis low selectivity as molecules are separated only by molecular size. In fact, 

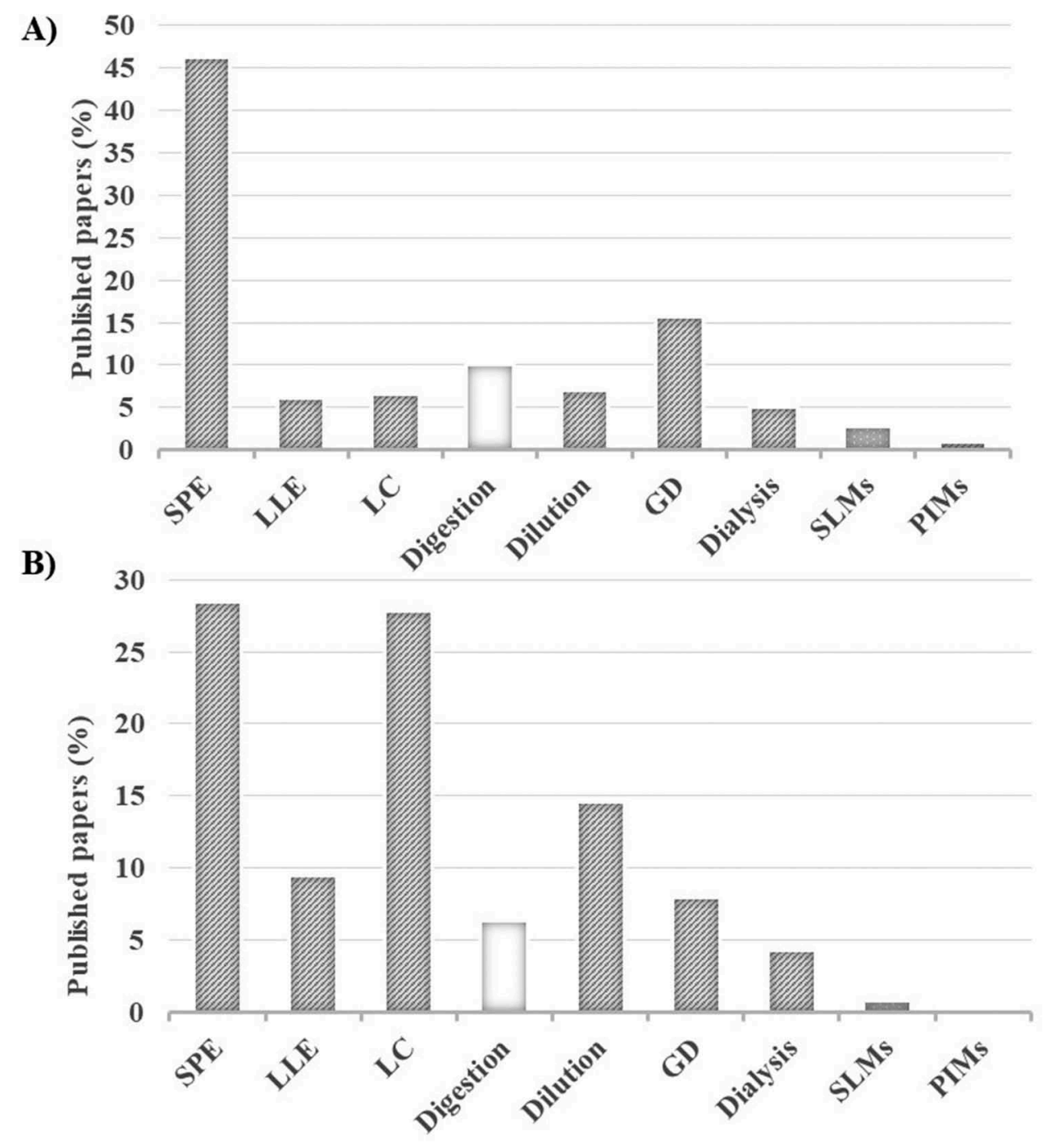

FIGURE 5. Distribution of the published papers by separation techniques used in hyphenation with: a) FIA and b) SIA (search made on ISI Web of Knowledge - Web of Science in October $25^{\text {th }} 2017$ ).

when using dialysis coupled to flow techniques, a decrease in sensitivity is usually observed (87). Similar to GDU, dialysis is also used in food analysis for matrix removal and sample dilution to fit high concentrations in the linear range of calibration curve therefore increasing the precision of the method. In the works by Silva, Álvares-Ribeiro, Oliveira et al. $(67,85)$ a dialysis unit was introduced in the flow system to attain sample dilution and minimize matrix interferences in wine analysis. Donnan dialysis has been scarcely used in combination with flow techniques in spite of providing high enrichment factors for cations probably due to the requirement of an ionic strength gradient. In the work by Antonia and Allen (86), Donnan dialysis was used for the determination of lead in spiked sweeteners and recoveries higher than $90 \%$ were obtained.
In the environmental field, dialysis can be used for the determination of analytes in samples without sample pretreatment or as an initial step for sample matrix removal prior to analyte pre-concentration removing particles that can clog the analytical column. Ganeshjeevan et al. (53) demonstrated the use of dialysis to separate $\mathrm{Cr}(\mathrm{VI})$ from organic matrices based on different molecular sizes. Additionally, dialysis can be used to protect flow-through electrodes and avoid their deterioration by avoiding direct contact of the sample with the active membrane of the sensor (81). Essentially, dialysis coupled to flow techniques is an effective tool for monitoring dynamic systems, a characteristic that could not be achieved using SPE or LLE.

Membrane extractions have been used as an alternative to LLE, since SLMs and PIMs use low or insignificant 


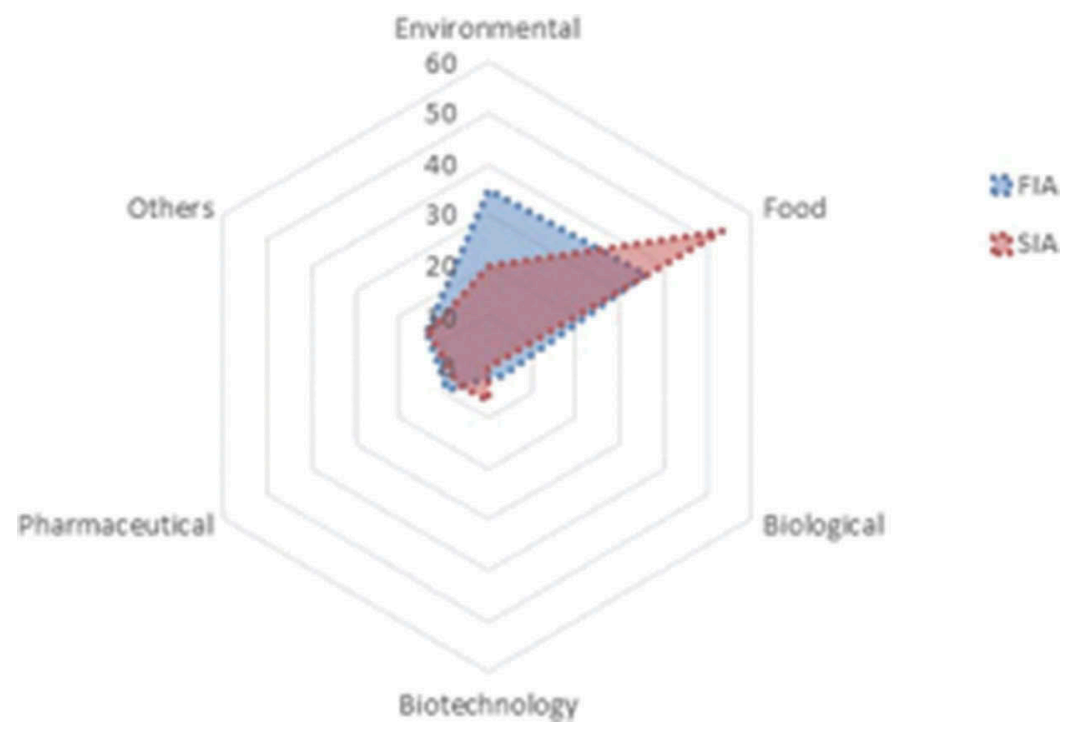

FIGURE 6. Applications of flow-based membrane extraction techniques (34-98).

amounts of organic reagents and are more easily coupled to flow techniques. When compared to LLE, SLM and PIM enable an easier automation of the sample preparation procedure without the need for large volume ratios between organic and aqueous phase to achieve high enrichment factors as required in LLE. Both SLMs and PIMs have been used for the determination of phenolic compounds, chromium (VI) and orthophosphate by molecular absorption spectroscopy, and for the determination of chlorinated phenols by capillary electrophoresis $(45,51,57,60)$ where the authors describe the use of SLMs for analyte enrichment.

Membrane-based separation techniques have been mainly used in FIA manifolds but they can also be employed in SIA. With SIA, a stopped flow can be more easily performed and the acceptor phase can be kept stopped to improve the diffusion through the membrane therefore increasing the diffusion efficiency and the pre-concentration capability. In the work by Butwong et al. (37), the acceptor stream was operated in a stopped-flow mode where a $60 \mathrm{~s}$ time was adopted to improve the sensitivity of the method. Kolev et al. (99) describe other approaches to improve mass transfer in on-line membranebased separation in flow analysis by flow manipulation. Approaches such as different fluid structures of the donor stream, stop-flow, oscillating flow and the introduction of air bubbles to separate the sample zone from the donor solution were tested. The authors concluded that flow manipulation in GD SIA using a second pump for the acceptor stream can improve the sensitivity of the method when compared to conventional GD FIA. This improvement was more pronounced for high molecular size analytes. Additionally, an improvement in sensitivity of 7-12.5-fold was obtained when both donor and acceptor streams were oscillated and the sample zone was separated by air bubbles from the donor solution. Some examples of flow configurations used to perform membrane-based separation are shown in Figure 7.

Figure 7a illustrates a SIA-SLM approach for in-line hollow-fiber (HF)-assisted three-phase liquid-phase microextraction (LPME). The developed method allowed the handling of the donor and acceptor aqueous solutions and of minimal volumes of the organic extracting phase in a programmable flow mode (57). Additionally, the hybrid flow analyzer for HF-LPME with in-line membrane regeneration allows sample clean-up through the removal of high molecular weight compounds and speciation analysis of $\mathrm{Cr}$ (VI) in troublesome harsh environmental samples and high organic load wastewaters.

In Figure 7b, a FIA-PIM approach is shown where the authors used a PVC/Aliquat 336 PIM to extract and preconcentrate reactive phosphate which was afterward determined by using the well-known molybdenum blue reaction (60). In the described work, the membrane extraction unit was placed in the sample loop of the injection valve which allowed the acceptor solution to be stopped during extraction. The flow system was run in a continuous flow mode unless pre-concentration was attended and, in this case, a 2min stopped period of time was adopted.

In SIA manifolds, the diffusion unit can be connected to the flow system in two different approaches: (a) the donor and the acceptor channels are connected to two different ports of the selection valve (Figure 7a, and c); (b) the donor channel connected to one port of the selection valve and the acceptor channel connected to a pump in a hybrid FIA-SIA manifold (Figure 7d).

The SIA-GD system shown in Figure 7c has been proposed for the determination of cyanides in tap and mineral water samples. In that work, the analyte is removed from 
SANTOS ET AL.

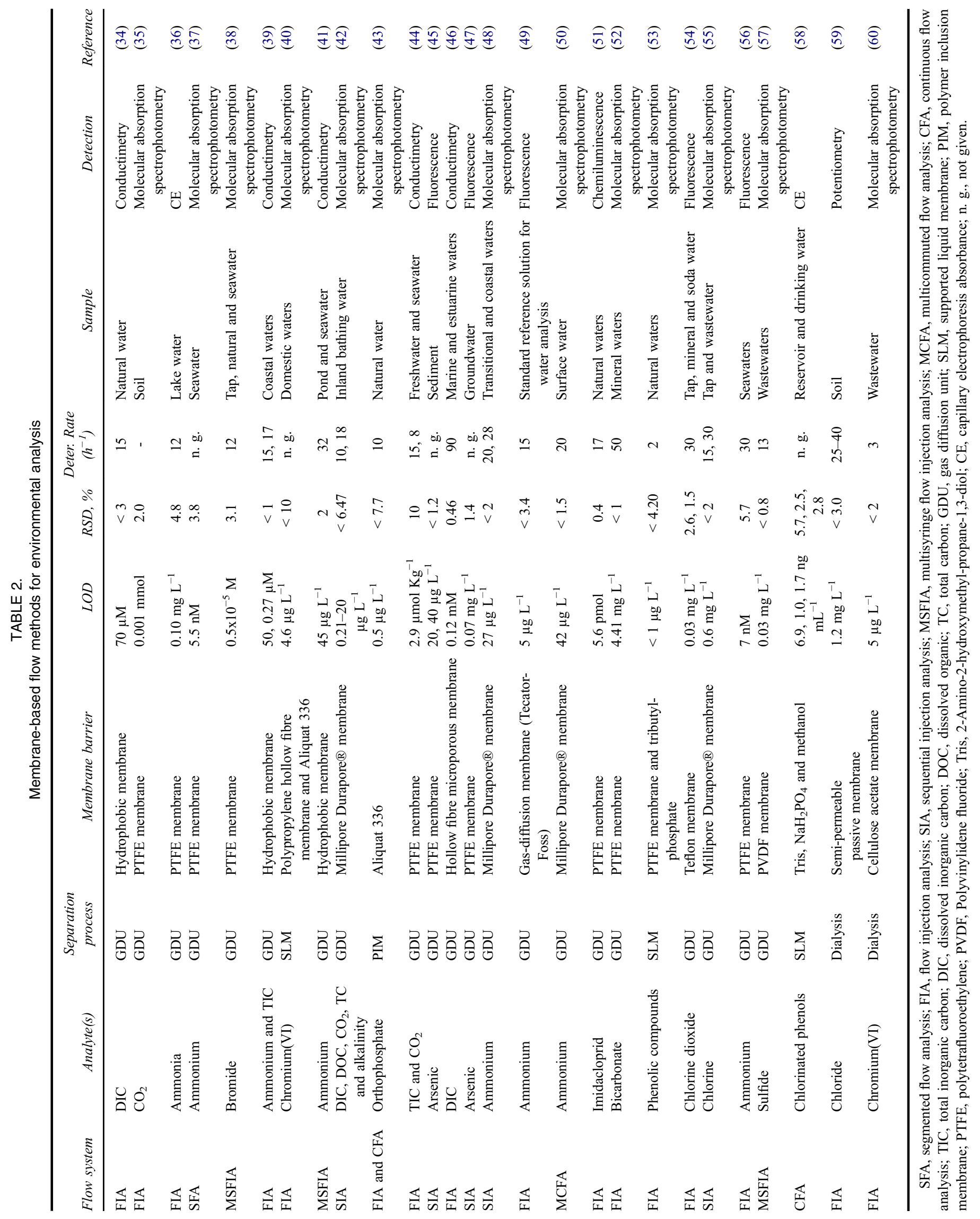




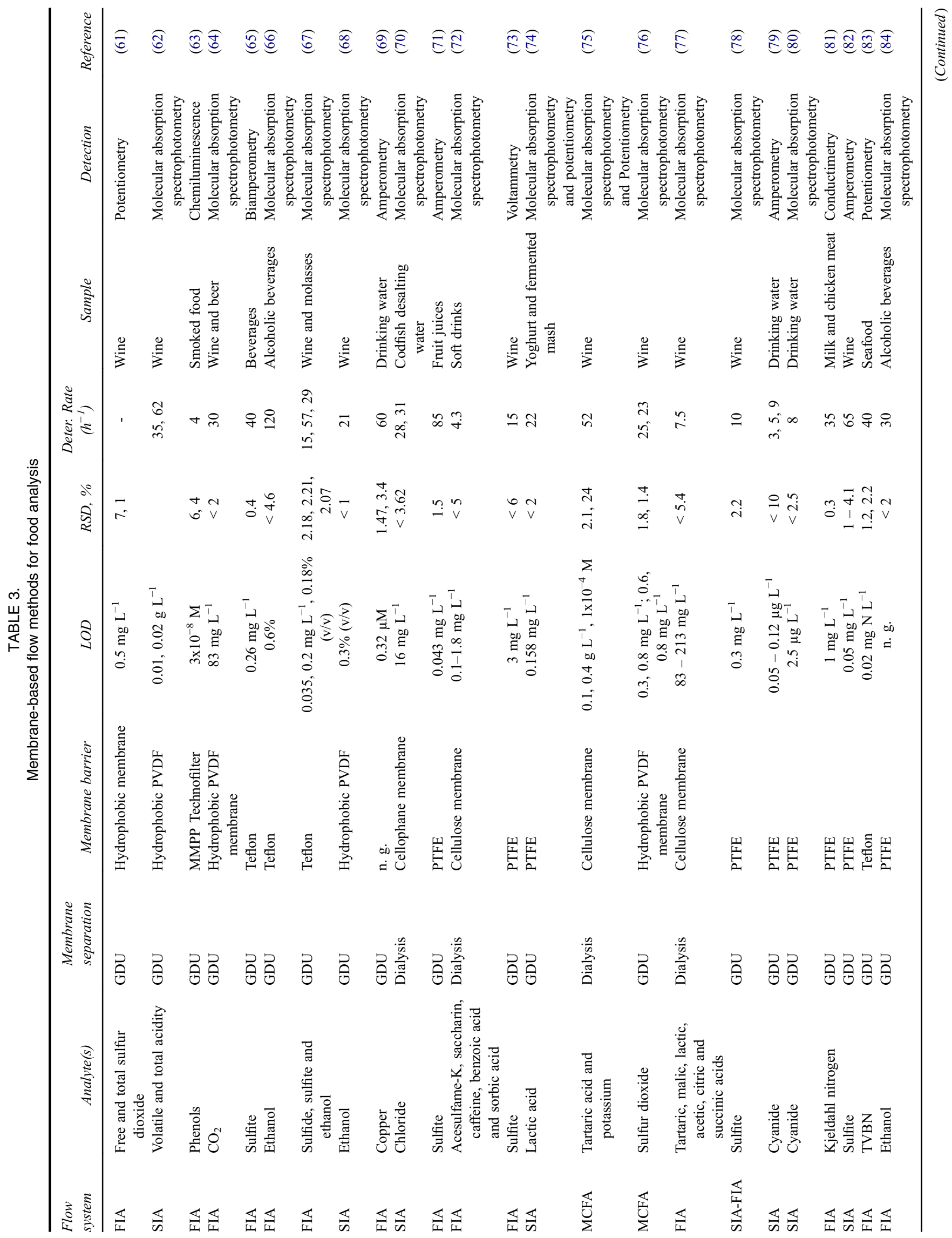




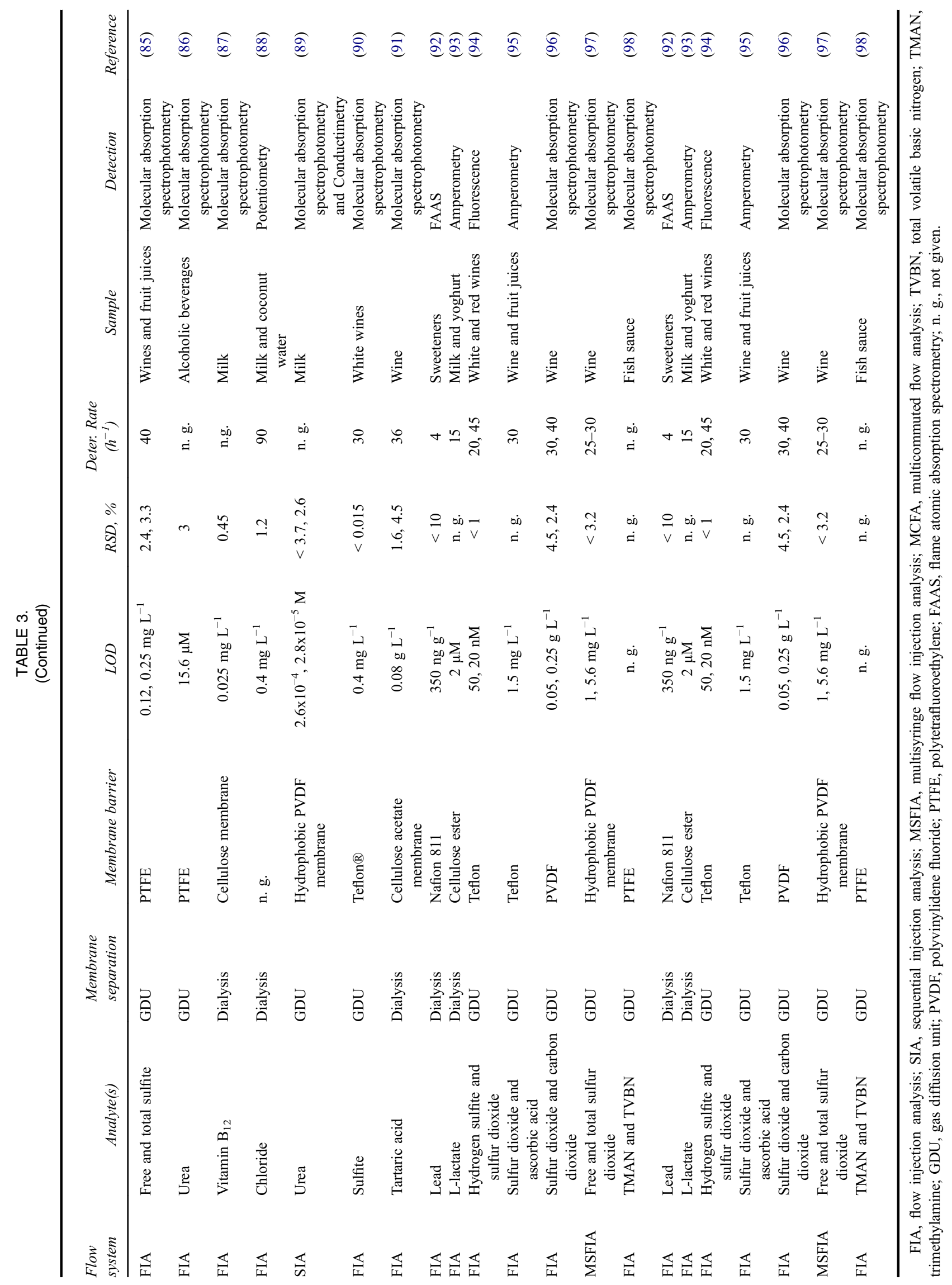


a)
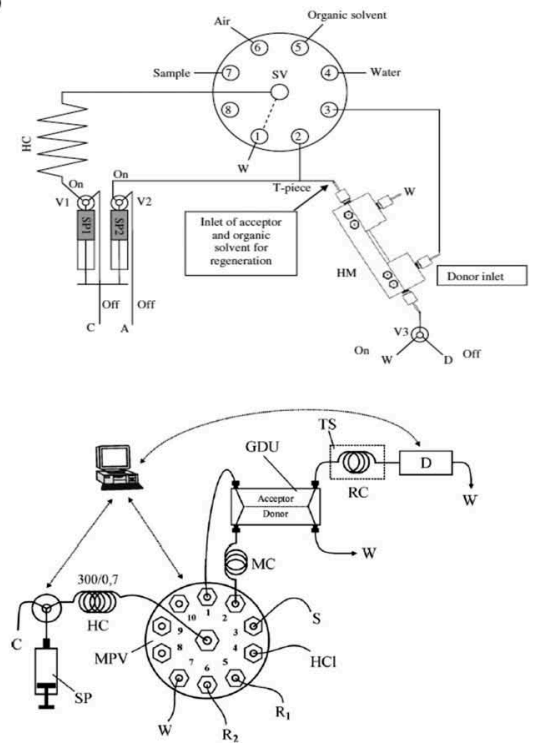
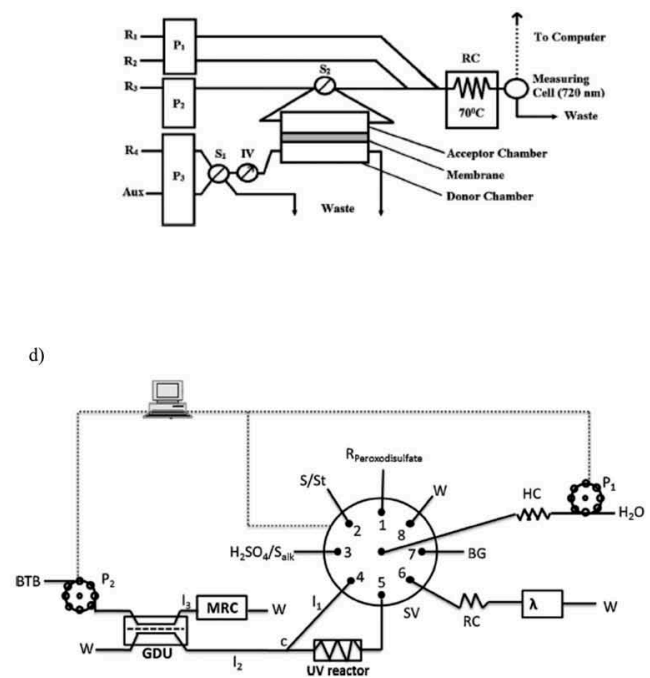

FIGURE 7. Schematic representations of FIA and SIA manifolds for membrane-based separations (a) SIA-SLM (Reprinted from Nitiyanontakit et al. (57) with permission from Springer. Copyright 2013); (b) FIA-PIM (Reprinted from Nagul et al. (60) with permission from Elsevier. Copyright 2013); (c) and (d) SIA-GDU (Reprinted from Themelis et al. (72) with permission from Elsevier. Copyright 2009 and Santos et al. (59) with permission from Elsevier. Copyright 2013, respectively).

sample matrix by acidification and diffusion through a gasdiffusion step. In the acceptor channel, cyanide reacts with ninhydrin in carbonate medium to form a colored product measured by spectrophotometry (72). The SIA-GD system depicted in Figure $7 \mathrm{~d}$ has been used in a FIA-SIA approach developed for the spectrophotometric determination of inorganic and organic carbon in water (59). An acid solution is added in-line to convert the dissolved inorganic carbon into carbon dioxide allowing its selective separation in the GDU and determination by changing the color of a $\mathrm{pH}$ indicator.

Overall, when combined with mass separation unit devices, flow techniques are mainly used to introduce the sample in the carrier stream where the analyte is derivatized for an enhanced diffusion. Afterward, the sample is propelled to the donor channel where mass transfer occurs. The extract can therefore be propelled to a detector or to a preconcentration unit for further enrichment. In fact, considering chromatographic techniques, membrane-based separation techniques can be a good front end, as they reduce the need for off-line sample pre-treatment. For example, in the works by Kritsunankul, Jakmunee et al. $(64,69)$, dialysis was used for sample pre-treatment in order to separate the analytes, food additives and organic acids, from the sample matrix before separation by HPLC.

Membrane-based separation techniques present several advantages namely the ease of automation and on-line hyphenation with flow-based techniques. Additionally, a high degree of sample cleanup and potential selectivity is attained which is an important improvement when compared to other sample preparation techniques such as SPE. In fact, in membrane extraction the analyte is intentionally transferred through a membrane while in SPE the analyte and other potential interferences can be adsorbed and released during elution (20). Nevertheless, when applied to complex sample matrices such as environmental and food samples, solid membranes can clog or rupture due to, for example, surfactants, which alters the analyte diffusion ability. Therefore, calibration curves should be performed on daily basis to confirm the membrane physical conditions.

The parameters mainly determined in environmental and food samples using membrane separation techniques coupled to flow analysis, are presented in Figure 8. As it would be expected, although SLMs can be very useful and used for inorganic ionic species, organic species are the most target analytes separated with these types of membranes. In methods using GD separation, inorganic species easily converted to gaseous form at room temperature are the targeted analytes. When combining dialysis with flow analysis, metal ions and inorganic species can be separated from other high molecular compounds.

More recently, liquid membranes have been used for sorptive microextraction. The work by Oshima et al. (100) describes the use of a packed column coated with a PIM as a sorbent for the on-line pre-concentration of thiocyanate in FIA. Since PIMs can be prepared according to the intended analyte, it is expected that these PIM coated columns can be used for the determination of a large range of analytes. 


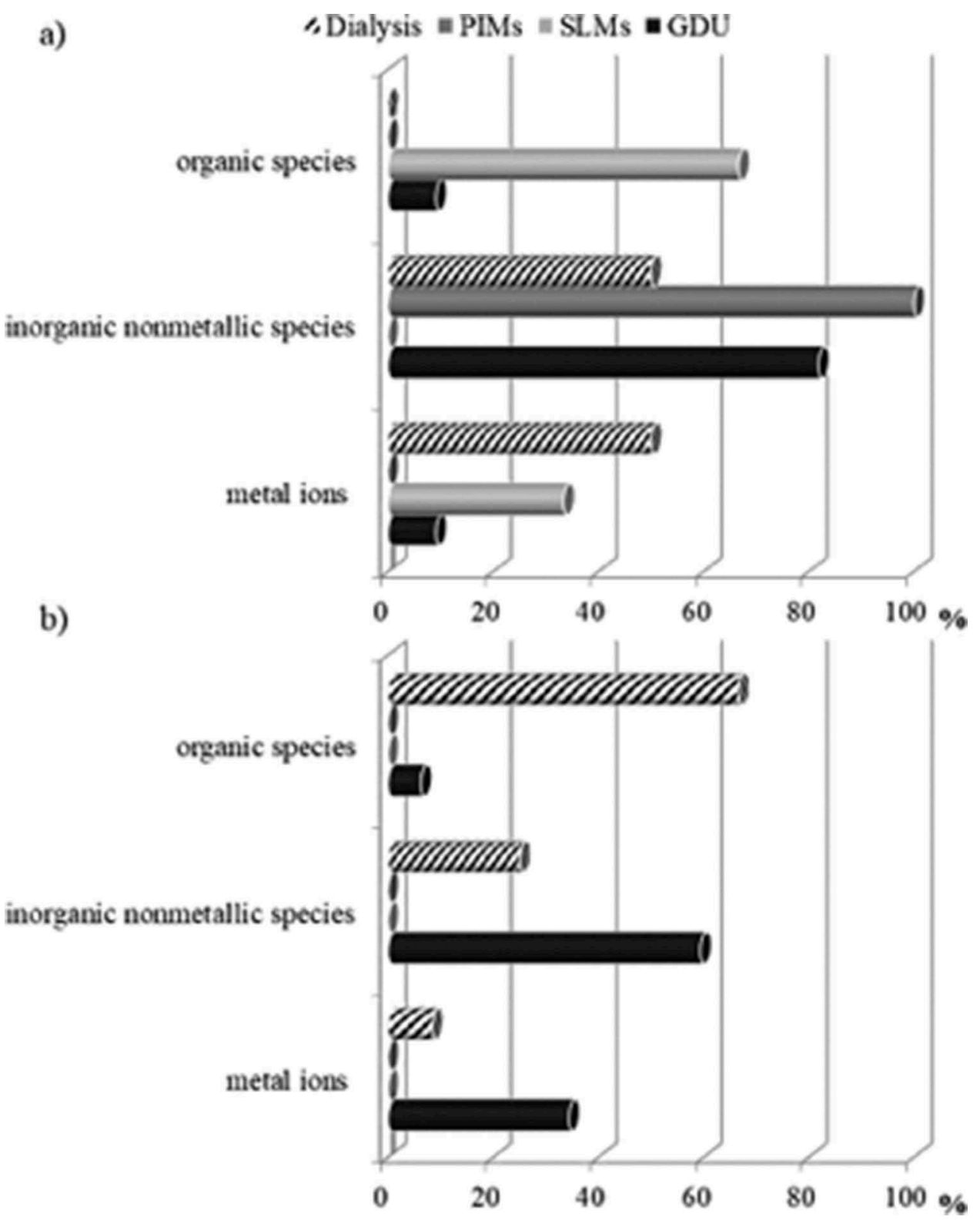

FIGURE 8. Distribution of the analytes targeted in the papers listed in Table 2 (34-60) and 3 (61-98) for (a) environmental applications; and (b) for food analysis.

\section{CHALLENGES}

Membrane-based separations present many advantages specially when coupled to flow analysis techniques. However, some challenges are still encountered. These methods suffer from a lack of specificity, permeability and resolution. Dialysis analyte recoveries rarely exceed 15-20\%. The extent to which continuous separation techniques enhance sensitivity depends on the volume ratio for the two liquids involved. Nowadays the needs for sensitive, specific and faster analytical methodologies are replacing these membrane separation approaches by other types of sample preparation. Compared to SPE and LLE, membrane separations are slower and have lower enrichment capacities. Due to this lower concentration ability, detection limits are usually higher which may require subsequent sample treatment. Additionally, membrane-based separation methods have lower throughput. Nevertheless, there has been an effort to adapt 96-well plate systems for membrane technologies. In this way, membrane techniques can become more competitive regarding their throughput when compared to other sample preparation techniques since all 96 samples can be processed simultaneously. Regarding flow analysis, 
several devices can be coupled in parallel to allow the simultaneous preparation of different samples.

Another disadvantage of membrane-separation is fouling: the membrane pores can be clogged when complex samples such as wastewater are analyzed. Furthermore, attention needs to be given to possible interactions between the analytes and the membrane to avoid lower recoveries and ensure method precision.

\section{CONCLUSIONS}

The use of membrane-based separation techniques in flow analysis for environmental and food applications provides valuable advantages such as the possibility to separate the analyte from complex sample matrices, analyte enrichment, reduction in organic reagents consumption, ease in operation and possibility of automation. Nevertheless, the efficiency of membrane separations is low when compared to other separation techniques, namely SPE resulting in a significant drawback. However, the yields of membrane-based methods can be optimized and adapted to the intended application (e.g., separation, enrichment or dilution). Still, when dialysis is coupled to flow injection techniques, the obtained yields are usually quite low and therefore this technique is mainly used for analyte dilution or sample clean-up. This may be one of the reasons for the low number of works describing the use of dialysis as an on-line sample pre-treatment.

Membrane extraction techniques have been a good alternative to LLE as these are more easily automated by flow techniques displaying lower consumption of organic reagents. Some of the works describe the use of this technique for analyte enrichment demonstrating the potential of this technique. GD is still the technique of choice in flow analysis as it allows the selective separation of compounds as only few are volatile at room temperature. Moreover, due to its selectivity, non-selective and cheaper detectors can be used. Additionally, membrane extraction techniques can be a good choice for a preliminary online sample pre-treatment (matrix cleanup or analyte enrichment) for chromatography.

In order to improve the membrane separation specificity, more work needs to be done in the development of new membranes to improve selectivity and permeability. Moreover, the use of PIMs for solid phase extraction must potentially be more explored as a cheaper alternative to SPE resins.

\section{ABBREVIATIONS}

FIA flow injection analysis

GD gas diffusion

GDU gas diffusion units

LLE liquid-liquid extraction

MCFA multicommuted flow analysis
MESI membrane extraction with a sorbent interface

MMLLE microporous membrane liquid-liquid extraction

MPFS multipumping flow systems

MSFIA multisyringe flow injection analysis

NAD + nicotinamide adenine dinucleotide

PIMs polymer inclusion membranes

PME polymeric membrane extraction

SIA sequential injection analysis

SLME supported liquid membrane extraction

SPE solid-phase extraction

$\mu$ SI-LOV micro-sequential injection lab-on-valve

\section{ACKNOWLEDGMENTS}

R.B.R. Mesquita thanks to Fundação para a Ciência e a Tecnologia (FCT) and POCH of FSE for the grant SFRH/ BPD/112032/2015.

\section{CONFLICT OF INTEREST}

There is no known conflict of interest.

\section{REFERENCES}

1. Raynie, D.E. (2016) Trends in sample preparation. $L C$-GC, 29: 142-154.

2. Pawliszyn, J. (2003) Sample preparation: quo vadis? Anal. Chem., 75: 2543-2558. doi:10.1021/ac034094h

3. Liska, I. (2000) Fifty years of solid-phase extraction in water analysis - historical development and overview. J. Chromatogr. A, 885: 3-16. doi:10.1016/S0021-9673(99)01144-9

4. Chen, Y., Guo, Z., Wang, X. and Qiu, C. (2008) Sample preparation. J. Chromatogr. A, 1184: 191-219. doi:10.1016/j.chroma.2007.10.026

5. Hennion, M.C. (1999) Solid-phase extraction: method development, sorbents, and coupling with liquid chromatography. J. Chromatogr. A, 856: 3-54. doi:10.1016/S0021-9673(99)00832-8

6. Silvestre, C.I.C., Santos, J.L.M., Lima, J.L.F.C. and Zagatto, E.A.G. (2009) Liquid - liquid extraction in flow analysis: a critical review. Anal. Chim. Acta, 652: 54-65. doi:10.1016/j.aca.2009.05.042

7. Miró, M. and Frenzel, W. (2004) Automated membrane-based sampling and sample preparation exploiting flow-injection analysis. $\operatorname{Tr} A C$ Trends Anal. Chem., 23: 624-636. doi:10.1016/j.trac.2004.07.006

8. Pan, J., Zhang, C., Zhang, Z. and Li, G. (2014) Review of online coupling of sample preparation techniques with liquid chromatography. Anal. Chim. Acta, 815: 1-15. doi:10.1016/j.aca.2014.01.017

9. Karlberg, B. (1988) Flow injection extraction in theory and practice. Fresenius J. Anal. Chem., 329: 660-662. doi:10.1007/BF00624770

10. Smith, R.M. (2003) Before the injection - modern methods of sample preparation for separation techniques. J. Chromatogr. A, 1000: 3-27. doi:10.1016/S0021-9673(03)00511-9

11. Ruzicka, J. and Hansen, E.H. (1975) Flow injection analyses. Anal. Chim. Acta, 78: 145-157. doi:10.1016/S0003-2670(01)84761-9

12. Ruzicka, J. and Marshall, G.D. (1990) Sequential injection: a new concept for chemical sensors, process analysis and laboratory assays. Anal. Chim. Acta, 237: 329-343. doi:10.1016/S0003-2670(00)83937-9

13. Segundo, M.A. and Rangel, A.O.S.S. (2002) Flow analysis: a critical view of its evolution and perspectives. J. Flow Inject. Anal., 19: 3-8. 
14. Ruzicka, J. (2000) Lab-on-valve: universal microflow analyzer based on sequential and bead injection. Analyst, 125: 1053-1060.

15. Ivaska, A. and Ruzicka, J. (1993) From flow injection to sequential injection: comparison of methodologies and selection of liquid drives. Analyst, 118: 885-889. doi:10.1039/AN9931800885

16. Ruzicka, J. and Hansen, E.H. (2008) Retro-review of flow-injection analysis. TrAC Trends Anal. Chem., 27: 390-393. doi:10.1016/j. trac.2008.03.004

17. Santos, I.C., Mesquita, R.B.R. and Rangel, A.O.S.S. (2015) The state of the art of flow-through solid-phase spectrometry. LC-GC North Am., 33: 25-31.

18. Gonçalves, L.M., Valente, I.M. and Rodrigues, J.A. (2017) Recent advances in membrane-aided extraction and separation for analytical purposes. Sep. Purif. Rev., 46: 179-194. doi:10.1080/ 15422119.2016.1235050

19. Frenzel, W. and Markeviciute, I. (2017) Membrane-based sample preparation for ion chromatography - techniques, instrumental configurations and applications. J. Chromatogr. A, 1479: 1-19. doi:10.1016/j.chroma.2016.11.052

20. Jönsson, J.Å. and Mathiasson, L. (2000) Membrane-based techniques for sample enrichment. J. Chromatogr. A, 902: 205-225. doi:10.1016/ S0021-9673(00)00922-5

21. Van De Merbel, N.C. (1999) Membrane-based sample preparation coupled on-line to chromatography or electrophoresis. $J$. Chromatogr. A, 856: 55-82. doi:10.1016/S0021-9673(99)00581-6

22. Cordero, B.M., Pavón, J.L.P., Pinto, C.G., Laespada, M.E.F., Martinez, R.C. and Gonzalo, E.R. (2000) Analytical applications of membrane extraction in chromatography and electrophoresis. $J$. Chromatogr. A, 902: 195-204. doi:10.1016/S0021-9673(00)00835-9

23. Staden, J.F. (1995) Membrane separation in flow injection systems. Fresenius. J. Anal. Chem., 352: 271-302. doi:10.1007/BF00322225

24. Miró, M. and Frenzel, W. (2005) The potential of microdialysis as an automatic sample-processing technique for environmental research. TrAC - Trends Anal. Chem., 24: 324-333. doi:10.1016/j. trac.2004.10.004

25. De Castro, M.D.L., Capote, F.P. and Ávila, N.S. (2008) Is dialysis alive as a membrane-based separation technique? TrAC Trends Anal. Chem., 27: 315-326. doi:10.1016/j.trac.2008.01.015

26. Pyrzynska, K. (2006) Preconcentration and recovery of metal ions by donnan dialysis. Microchim. Acta, 153: 117-126. doi:10.1007/ s00604-005-0434-4

27. Moskvin, L.N. and Nikitina, T.G. (2004) Membrane methods of substance separation in analytical chemistry. J. Anal. Chem., 59: 2-16.

28. Sastre, A.M., Kumar, A., Shukla, J.P. and Singh, R.K. (1998) Improved techniques in liquid membrane separations: an overview. Sep. Purif. Methods, 27: 213-298. doi:10.1080/03602549809351641

29. Nghiem, L.D., Mornane, P., Potter, I.D., Perera, J.M., Cattrall, R.W. and Kolev, S.D. (2006) Extraction and transport of metal ions and small organic compounds using polymer inclusion membranes (PIMs). J. Memb. Sci., 281: 7-41. doi:10.1016/j.memsci.2006.03.035

30. Almeida, G.S., Cattrall, R.W. and Kolev, S.D. (2017) Polymer inclusion membranes (PIMs) in chemical analysis - a review. Anal. Chim. Acta, 987: 1-14. doi:10.1016/j.aca.2017.07.032

31. Kolev, S. and McKelvie, I. (2008) Advances in Flow Injection Analysis and Related Techniques; Comprehensive Analytical Chemistry; Elsevier Science: New York, USA.

32. Cerdá, V. (2014) Flow Analysis: A Practical Guide; Elsevier Science: New York, USA.

33. Fialab Systems. Sandwich gas membrane sensor. http://www.flowin jection.com/index.php/products/flow-cells?pid=61\&sid=100: sand wich-membrane-flow-cell-smfc (accessed May 3, 2018).

34. Somboot, W., Jakmunee, J. and Kanyanee, T. (2017) An exploiting of cost-effective direct current conductivity detector in gas diffusion flow injection system. Talanta, 170: 298-305. doi:10.1016/j. talanta.2017.04.015
35. Silva, C.R., Oliveira, E., Zagatto, E.A.G. and Henriquez, C. (2016) A novel flow-based procedure for automation of respirometric assays in soils. Talanta, 158: 14-20. doi:10.1016/j.talanta.2016.05.026

36. Martinotti, V., Balordi, M. and Ciceri, G. (2012) A flow injection analyser conductometric coupled system for the field analysis of free dissolved $\mathrm{CO} 2$ and total dissolved inorganic carbon in natural waters. Anal. Bioanal. Chem., 403: 1083-1093. doi:10.1007/s00216-012$5762-8$

37. Butwong, N., Srijaranai, S., Ngeontae, W. and Burakham, R. (2012) Speciation of arsenic (III) and arsenic (V) based on quenching of CdS quantum dots fluorescence using hybrid sequential injection-stopped flow injection gas-diffusion system. Spectrochim. Acta Part A Mol. Biomol. Spectrosc., 97: 17-23. doi:10.1016/j.saa.2012.05.054

38. Pencharee, S., Faber, P.A., Ellis, P.S., Cook, P., Intaraprasert, J., Grudpan, K. and Mckelvie, I. (2012) Underway determination of dissolved inorganic carbon in estuarine waters by gas-diffusion flow analysis with C4D detection. Anal. Methods, 4: 1278-1283. doi:10.1039/c2ay25113b

39. Butwong, N., Noipa, T., Burakham, R., Srijaranai, S. and Ngeontae, W. (2011) Determination of arsenic based on quenching of CdS quantum dots fluorescence using the gas-diffusion flow injection method. Talanta, 8: 1063-1069. doi:10.1016/j.talanta.2011.05.023

40. Segundo, R.L.A., Mesquita, R.B.R., Ferreira, M.T.S.O.B., Teixeira, C. F.C.P., Bordalo, A.A. and Rangel, A.O.S.S. (2011) Development of a sequential injection gas diffusion system for the determination of ammonium in transitional and coastal waters. Anal. Methods, 3: 2049-2055. doi:10.1039/c1ay05129f

41. Almendral-Parra, M.-J., Alonso-Mateos, A. and Fuentes-Prieto, M.-S (2010) A gas diffusion technique coupled with flow injection systems. Optimization of the process in its application to the fluorimetric determination of ammonium in water samples. J. Fluoresc., 20: 5565. doi:10.1007/s10895-009-0521-8

42. Oliveira, S.M., Lopes, T.I.M.S., Tóth, I.V. and Rangel, A.O.S.S. (2007) A multi-commuted flow injection system with a multi-channel propulsion unit placed before detection: spectrophotometric determination of ammonium. Anal. Chim. Acta, 600: 29-34. doi:10.1016/j. aca.2007.01.019

43. Lagalante, A.F. and Greenbacker, P.W. (2007) Flow injection analysis of imidacloprid in natural waters and agricultural matrixes by photochemical dissociation, chemical reduction, and nitric oxide chemiluminescence detection. Anal. Chim. Acta, 590: 151-158. doi:10.1016/j. aca.2007.03.034

44. Oliveira, P.C.C., Masini, J.C., Galhardo, C.X., Lima, J.C.S., Sant'ana, A.E.G., Vasconcelos, A.M.G., Nunes, W.P. and Amaral, O.L.C. (2006) A new approach to construct diffusion/permeation cell for use in flow systems. Application in the spectrophotometric determination of bicarbonate ions. J. Braz. Chem. Soc., 17: 976-980. doi:10.1590/S0103-50532006000500023

45. Sun, A., Li, J. and Liu, R. (2006) High-performance liquid chromatographic determination of phenolic compounds in natural water coupled with on-line flow injection membrane extraction-preconcentration. J. Sep. Sci., 29: 995-1000.

46. Zhu, Z., Lu, J.J., Almeida, M.I.G.S., Pu, Q., Kolev, S.D. and Liu, S. (2015) A microfabricated electroosmotic pump coupled to a gas-diffusion microchip for flow injection analysis of ammonia. Microchim. Acta, 182: 1063-1070. doi:10.1007/s00604-0141410-7

47. Themelis, D.G. and Kika, F.S. (2006) Gas-diffusion flow injection assay for the selective determination of chlorine dioxide based on the fluorescence quenching of chromotropic acid. Microchem. J., 82: 108-112. doi:10.1016/j.microc.2005.12.001

48. Mesquita, R.B.R. and Rangel, A.O.S.S. (2005) Gas diffusion sequential injection system for the spectrophotometric determination of free chlorine with o-dianisidine. Talanta, 68: 268-273. doi:10.1016/j. talanta.2005.07.028 
49. Watson, R.J., Butler, E.C.V., Clementson, L.A. and Berry, K.M. (2005) Flow-injection analysis with fluorescence detection for the determination of trace levels of ammonium in seawater. J. Environ. Monit., 7: 37-42. doi:10.1039/b405924g

50. De Armas, G., Ferrer, L., Miró, M., Estela, J.M. and Cerdà, V. (2004) In-line membrane separation method for sulfide monitoring in wastewaters exploiting multisyringe flow injection analysis. Anal. Chim. Acta, 524: 89-96. doi:10.1016/j.aca.2004.02.050

51. Zhou, Q., Liu, J., Jiang, G., Liu, G. and Cai, Y. (2004) Sensitivity enhancement of chlorinated phenols by continuous flow liquid membrane extraction followed by capillary electrophoresis. J. Sep. Sci., 27: 576-580. doi:10.1002/jssc.200301632

52. Miró, M. and Frenzel, W. (2003) A novel flow-through microdialysis separation unit with integrated differential potentiometric detection for the determination of chloride in soil samples. Analyst, 128: 12911297. doi:10.1039/B306747E

53. Ganeshjeevan, R., Chandrasekar, R., Yuvaraj, S. and Radhakrishnan, G. (2003) Determination of hexavalent chromium by on-line dialysis ion chromatography in a matrix of strong colourants and trivalent chromium. J. Chromatogr. A, 988: 157-165. doi:10.1016/S0021-9673 (02)02051-4

54. Kodama, T., Ichikawa, T., Hidaka, K. and Furuya, K. (2015) A highly sensitive and large concentration range colorimetric continuous flow analysis for ammonium concentration. J. Oceanogr., 71: 65-75. doi:10.1007/s10872-014-0260-6

55. Danchana, K., Maya, F., Wilairat, P., Uraisin, K. and Cerdà, V. (2015) Spectrophotometric determination of bromide in water using the multisyringe flow injection analysis technique coupled to a gas-diffusion unit. Anal. Methods, 7: 4202-4208. doi:10.1039/C5AY00202H

56. Henriquez, C., Horstkotte, B. and Cerdà, V. (2014) A highly reproducible solenoid micropump system for the analysis of total inorganic carbon and ammonium using gas-diffusion with conductimetric detection. Talanta, 118: 186-194. doi:10.1016/j.talanta.2013.10.005

57. Nitiyanontakit, S., Varanusupakul, P. and Miró, M. (2013) Hybrid flow analyzer for automatic hollow-fiber-assisted ionic liquid-based liquidphase microextraction with in-line membrane regeneration. Anal. Bioanal. Chem., 405: 3279-3288. doi:10.1007/s00216-013-6744-1

58. Henríquez, C., Horstkotte, B. and Cerdà, V. (2013) Conductometric determination of ammonium by a multisyringe flow injection system applying gas diffusion. Int. J. Environ. Anal. Chem., 93: 1236-1252. doi:10.1080/03067319.2012.746322

59. Santos, I.C., Mesquita, R.B.R., Machado, A., Bordalo, A.A. and Rangel, A.O.S.S. (2013) Sequential injection methodology for carbon speciation in bathing waters. Anal. Chim. Acta, 778: 38-47. doi:10.1016/j.aca.2013.03.043

60. Nagul, E.A., Fontàs, C., McKelvie, I.D., Cattrall, R.W. and Kolev, S. D. (2013) The use of a polymer inclusion membrane for separation and preconcentration of orthophosphate in flow analysis. Anal. Chim. Acta, 803: 82-90. doi:10.1016/j.aca.2013.07.052

61. Giménez-Gómez, P., Gutiérrez-Capitán, M., Puig-Pujol, A., Capdevila, F., Muñoz, S., Tobeña, A., Miró, A. and JiménezJorquera, C. (2017) Analysis of free and total sulfur dioxide in wine by using a gas-diffusion analytical system with $\mathrm{pH}$ detection. Food Chem., 228: 518-525. doi:10.1016/j.foodchem.2017.02.026

62. Vidigal, S.S.M.P. and Rangel, A.O.S.S. (2017) A flow-based platform for measuring the acidity parameters in wine. Talanta, 168: 313-319. doi:10.1016/j.talanta.2017.03.029

63. Martins, P.R., Popolim, W.D., Nagato, L.A.F., Takemoto, E., Araki, K., Toma, H.E., Angnes, L. and Penteado, M.D.V.C. (2011) Fast and reliable analyses of sulphite in fruit juices using a supramolecular amperometric detector encompassing in flow gas diffusion unit. Food Chem., 127: 249-255. doi:10.1016/j.foodchem.2010.12.103

64. Kritsunankul, O. and Jakmunee, J. (2011) Simultaneous determination of some food additives in soft drinks and other liquid foods by flow injection on-line dialysis coupled to high performance liquid chromatography. Talanta, 84: 1342-1349. doi:10.1016/j.talanta.2011.02.045

65. Gonçalves, L.M., Pacheco, J.G. and Magalhães, P.J. (2010) Determination of free and total sulfites in wine using an automatic flow injection analysis system with voltammetric detection. Food Addit. Contam. Part A, 27: 175-180. doi:10.1080/ 19440040903261547

66. Dias, A.C.B., Silva, R.A.O. and Arruda, M.A.Z. (2010) A sequential injection system for indirect spectrophotometric determination of lactic acid in yogurt and fermented mash samples. Microchem. J., 96: 151-156. doi:10.1016/j.microc.2010.02.016

67. Oliveira, S.M., Lopes, T.I.M.S., Tóth, I.V. and Rangel, A.O.S.S. (2010) Simultaneous determination of tartaric acid and potassium in wines using a multicommuted flow system with dialysis. Talanta, 81: 1735-1741. doi:10.1016/j.talanta.2010.03.032

68. Oliveira, S.M., Lopes, T.I.M.S., Tóth, I.V. and Rangel, A.O.S.S. (2009) Development of a gas diffusion multicommuted flow injection system for the determination of sulfur dioxide in wines, comparing malachite green and pararosaniline chemistries. J. Agric. Food Chem., 57: 3415-3422. doi:10.1021/jf803639n

69. Kritsunankul, O., Pramote, B. and Jakmunee, J. (2009) Flow injection on-line dialysis coupled to high performance liquid chromatography for the determination of some organic acids in wine. Talanta, 79: 1042-1049. doi:10.1016/j.talanta.2009.03.001

70. Tzanavaras, P.D., Thiakouli, E. and Themelis, D.G. (2009) Hybrid sequential injection - flow injection manifold for the spectrophotometric determination of total sulfite in wines using o-phthalaldehyde and gas-diffusion. Talanta, 77: 1614-1619. doi:10.1016/j. talanta.2008.07.055

71. Zacharis, C.K., Tzanavaras, P.D., Voulgaropoulos, A.N. and Karlberg, B. (2009) Amperometric determination of cyanides at the low ppb level by automated preconcentration based on gas diffusion coupled to sequential injection analysis. Talanta, 77: 1620-1626. doi:10.1016/j. talanta.2008.07.055

72. Themelis, D.G., Karastogianni, S.C. and Tzanavaras, P.D. (2009) Selective determination of cyanides by gas diffusion-stopped flowsequential injection analysis and an on-line standard addition approach. Anal. Chim. Acta, 632: 93-100. doi:10.1016/j. aca.2008.10.074

73. Vakh, C., Evdokimova, E., Pochivalov, A., Moskvin, L. and Bulatov, A. (2017) A novel flow injection chemiluminescence method for automated and miniaturized determination of phenols in smoked food samples. Food Chem., 237: 929-935. doi:10.1016/j. foodchem.2017.06.049

74. Junsomboon, J. and Jakmunee, J. (2008) Flow injection conductometric system with gas diffusion separation for the determination of Kjeldahl nitrogen in milk and chicken meat. Anal. Chim. Acta, 627: 232-238. doi:10.1016/j.aca.2008.08.012

75. Chinvongamorn, C., Pinwattana, K., Praphairaksit, N., Imato, T. and Chailapakul, O. (2008) Amperometric determination of sulfite by gas diffusion-sequential injection with boron-doped diamond electrode. Sensors, 8: 1846-1857. doi:10.3390/s8010464

76. Dhaouadi, A., Monser, L. and Sadok, S. (2007) Validation of a flowinjection-gas diffusion method for total volatile basic nitrogen determination in seafood products. Food Chem., 103: 1049-1053. doi:10.1016/j.foodchem.2006.07.066

77. Vicente, S., Zagatto, E.A.G. and Borges, E.P. (2006) Exploiting gas diffusion for non-invasive sampling in flow analysis: determination of ethanol in alcoholic beverages. An. Acad. Bras. Cienc., 78: 23-29. doi:10.1590/S0001-37652006000100004

78. Carinhanha, J., Santos, C. and Korn, M. (2006) Exploiting sulphide generation and gas diffusion separation in a flow system for indirect sulphite determination in wines and fruit juices. Microchim. Acta, 153: 87-94. doi:10.1007/s00604-005-0453-1 
79. Iida, Y., Suganuma, Y., Matsumoto, K. and Satoh, I. (2006) Novel determination system for urea in alcoholic beverages by using an FIA system with an acid urease column. Anal. Sci., 22: 173-176. doi:10.2116/analsci.22.173

80. Medina-Alonso, G., Carrasco-Fuentes, M. and Canizares-Macías, M. P. (2005) Coupling on-line of a dialyser with a flow-continuous system to separate Vitamin B12 from milk. Talanta, 68: 292-297. doi:10.1016/j.talanta.2005.08.028

81. Da Silva, I.S., Richter, E.M., Do Lago, C.L., Gutz, I.G., Tanaka, A.A. and Angnes, L. (2005) FIA-potentiometry in the sub-Nernstian response region for rapid and direct chloride assays in milk and in coconut water. Talanta, 67: 651-657. doi:10.1016/j. talanta.2005.03.010

82. Lima, M.J.R., Fernandes, S.M.V. and Rangel, A.O.S.S. (2004) Enzymatic determination of urea in milk by sequential injection with spectrophotometric and conductometric detection. J. Agric. Food Chem., 52: 6887-6890. doi:10.1021/jf0488312

83. Melo, D., Zagatto, E.A.G., Mattos, I.L. and Maniasso, N. (2003) Spectrophotometric flow-injection determination of sulphite in white wines involving gas diffusion through a concentric tubular membrane. J. Braz. Chem. Soc., 14: 375-379. doi:10.1590/S010350532003000300006

84. Calvo-López, A., Ymbern, O., Izquierdo, D. and Alonso-Chamarro, J. (2016) Low cost and compact analytical microsystem for carbon dioxide determination in production processes of wine and beer. Anal. Chim. Acta, 931: 64-69. doi:10.1016/j.aca.2016.05.010

85. Silva, H.A.D.F.O. and Álvares-Ribeiro, L.M.B.C. (2002) Optimization of a flow injection analysis system for tartaric acid determination in wines. Talanta, 58: 1311-1318. doi:10.1016/S0039-9140(02)00436-8

86. Antonia, A. and Allen, L.B. (2001) Extraction and analysis of lead in sweeteners by flow-Injection donnan dialysis with flame atomic absorption spectroscopy. J. Agric. Food Chem., 49: 4615-4618. doi:10.1021/jf010504w

87. Palmisano, F., Quinto, M., Rizzi, R. and Zambonin, P.G. (2001) Flow injection analysis of L-lactate in milk and yoghurt by on-line microdialysis and amperometric detection at a disposable biosensor. Analyst, 126: 866-870. doi:10.1039/b010180j

88. Mana, H. and Spohn, U. (2001) Sensitive and selective flow injection analysis of hydrogen sulfite/sulfur dioxide by fluorescence detection with and without membrane separation by gas diffusion. Anal. Chem., 73: 3187-3192. doi:10.1021/ac001049q

89. Cardwell, T.J. and Christophersen, M.J. (2000) Determination of sulfur dioxide and ascorbic acid in beverages using a dual channel flow injection electrochemical detection system. Analyst, 416: 105-110.

90. Atanassov, G.T., Lima, R.C., Mesquita, R.B.R., Rangel, A.O.S.S. and Tóth, I.V. (2000) Spectrophotometric determination of carbon dioxide and sulphur dioxide in wines by flow injection. Analusis, 28: 77-82. doi:10.1051/analusis:2000100

91. Segundo, M.A., Rangel, A.O.S.S., Cladera, A. and Cerdà, V. (2000) Multisyringe flow system: determination of sulfur dioxide in wines. Analyst, 125: 1501-1505.

92. Ruiz-Capillas, C., Gillyon, C.M. and Horner, W.F.A. (2000) Determination of volatile basic nitrogen and trimethylamine nitrogen in fish sauce by flow injection analysis. Eur. Food Res. Technol., 210: 434-436. doi:10.1007/s002170050577

93. Paula, N.T.G., Barbosa, E.M.O., Da Silva, P.A.B., De Souza, G.C.S., Nascimento, V.B. and Lavorante, A.F. (2016) In-line electrochemical reagent generation coupled to a flow injection biamperometric system for the determination of sulfite in beverage samples. Food Chem., 203: 183-189. doi:10.1016/j.foodchem.2016.01.125

94. Vidigal, S.S.M.P. and Rangel, A.O.S.S. (2015) A reagentless flow injection system for the quantification of ethanol in beverages based on the schlieren effect measurement. Microchem. J., 121: 107-111. doi:10.1016/j.microc.2015.02.006

95. Silva, C.R., Gomes, T.F., Barros, V.A.F. and Zagatto, E.A.G. (2013) A multi-purpose flow manifold for the spectrophotometric determination of sulphide, sulphite and ethanol involving gas diffusion: application to wine and molasses analysis. Talanta, 113: 118-122. doi:10.1016/j. talanta.2013.03.021

96. Pais, T.F.M., Vidigal, S.S.M.P., Tóth, I.V. and Rangel, A.O.S.S. (2013) Sequential injection system for the enzymatic determination of ethanol in alcoholic beverages with in-line dilution. Food Control, 30: 616-620. doi:10.1016/j.foodcont.2012.08.013

97. Lolic, A., Tripkovic, T., Baosic, R., Nikolic-Mandic, S. and Stanimirovic, B. (2012) Development of flow injection method for indirect copper determination with amperometric detection in drinking water samples. J. Serbian Chem. Soc., 77: 1641-1647. doi:10.2298/ JSC120616090L

98. Santos, I.C., Mesquita, R.B.R., Galvis-Sanchez, A.C., Delgadillo, I. and Rangel, A.O.S.S. (2012) Development of a turbidimetric sequential injection system to monitor the codfish desalting process. Food Anal. Methods, 5: 287-295. doi:10.1007/s12161-0119238-9

99. Kolev, S.D., Fernandes, P.R.L.V., Satinsky, D. and Solich, P. (2009) Highly sensitive gas-diffusion sequential injection analysis based on flow manipulation. Talanta, 79: 1021-1025. doi:10.1016/j. talanta.2009.02.014

100. Ohshima, T., Kagaya, S., Gemmei-Ide, M., Cattrall, R.W. and Kolev, S.D. (2014) The use of a polymer inclusion membrane as a sorbent for online preconcentration in the flow injection determination of thiocyanate impurity in ammonium sulfate fertilizer. Talanta, 129: 560564. doi:10.1016/j.talanta.2014.06.029 